\title{
Cancer Research Group
}

National Cancer Institute

\section{Source}

National Cancer Institute. Cancer Research Group. NCI Thesaurus. Code C48168.

A collection of scientist and/or clinician users with a common research objective related to diagnosis and treatment of cancer. 